\title{
Intervenções de enfermagem para os diagnósticos de enfermagem mais freqüentes em dois serviços públicos de assistência à saúde da mulher*
}

\author{
Nursing interventions formulated for the most frequent nursing diagnoses \\ in gynecology at two regional health centers \\ Intervenciones de enfermería para los diagnósticos de enfermería más
frecuentes en ginecología en centros regionales de salud
}

Maria Auxiliadora de Souza Gerk', Sônia Maria Oliveira de Barros²

\section{RESUMO}

Introdução: Trata-se de uma pesquisa descritiva, analítica, retrospectiva sobre as intervenções de enfermagem para os diagnósticos de enfermagem mais freqüentes em ginecologia, entre as 165 mulheres assistidas em consultas de enfermagem em dois centros regionais de saúde de Campo Grande, MS, e sobre a construção destas intervenções a partir dos eixos A (tipo ação), B (alvo), C (meios) e H (beneficiário) da Classificação Internacional para a Prática de Enfermagem (CIPE ${ }^{\circledR}$ - versão beta 2. Objetivos: Estabelecer as intervenções de enfermagem e os resultados esperados. Método: Foram analisados 165 protocolos de consulta de enfermagem nas quais havia sido utilizado um instrumento de levantamento de dados sistematizado. Resultados: Foram identificados 60 diagnósticos de enfermagem, cuja freqüência simples variou de um a 137, dos quais selecionamos os cinco mais freqüentes para o estabelecimento das intervenções. As ações estabelecidas mais freqüentes foram 'orientar', 'referir', 'explicar' e 'prover'. Conclusões: Ao estabelecermos as intervenções, verificamos a necessidade premente do trabalho multidisciplinar, uma vez que a ação 'encaminhar' significou o equivalente ao encaminhamento a outros serviços de saúde. Outra constatação foi a imprescindibilidade do papel de educadora da enfermeira.

Descritores: Saúde da mulher; Enfermagem obstétrica; Diagnóstico de enfermagem

\begin{abstract}
Introduction: This study constitutes a descriptive, analytical, and retrospective research on the nursing interventions formulated for the most frequent nursing diagnoses in gynecology identified for 165 women seen at outpatient nursing appointments held at two regional health centers in Campo Grande, MS, Brazil. The interventions were developed from axes A (action type), B (target), C (means), and H (beneficiary) of the ICNP ${ }^{\circledR}$ Nursing Actions Classification (Beta 2 version). Objectives: Establish nursing interventions and nursing outcomes. Method: The data on the population were obtained from the protocols of 165 outpatient consultation sessions. Results: Sixty nursing diagnoses were identified, whose simple frequency ranged from 1 to 137, and the five most frequent ones were selected for establishing the nursing interventions. The actions most frequently established were 'guiding', 'referring', 'explaining', and 'providing'. Conclusions: While formulating the interventions, an urgent need for multidisciplinary work was perceived, considering that the action 'referring', in the scope of this research, equated the referral to diverse health care services. Another finding was the evidence that nurses play a pivotal role as education agents.
\end{abstract}

Keywords: Women's health; Obstetrical nursing; Nursing diagnosis

\section{RESUMEN}

Introducion: Se trata de una pesquisa descriptiva, analítica retrospectiva sobre las intervenciones de enfermería para los diagnósticos de enfermería más frecuentes en ginecología dentre las 165 mujeres asistidas en consultas de enfermería en dos centros regionales de salud de Campo Grande, MS, y sobre la construcción de estas intervenciones a partir de los ejes A (tipo acción), B (blanco), C (medios) y $\mathrm{H}$ (beneficiario) de la clasificación internacional para la práctica de enfermería, cuya frecuencia simple ha sido variada de uno a 137, de los cuales seleccionamos los cinco más frecuentes para el establecimiento de las intervenciones. Objetivos: Constucción de las intervenciones de enfermería Método: Foram analisados 165 protocolos de consulta de enfermagem nas quais havia sido utilizado um instrumento de levantamento de dados sistematizado. Resultados: Las acciones establecidas más frecuentes fueron 'orientar', 'referir 'explicar y provenir. Conclusión: Determinando las intervenciones, verificamos la extrema necesidad del trabajo multidisciplinar, pues la acción "direccionar" ha significado, en nuestra pesquisa, lo equivalente al direccionamiento a otros campos de salud. Y además de eso ha sido constatado la importancia de la enfermera como educadora.

Descriptores: Salud de las mujeres; Enfermería obstétrica; Diagnostico de enfermería

Trabalho extraído da Tese de Doutorado "Saúde da Mulher: Intervenções de Enfermagem em Ginecologia", apresentada à Universidade Federal de São Paulo - UNIFESP - São Paulo (SP), Brasil.

1 Professora assistente do Departamento de Enfermagem da Universidade Federal de Mato Grosso do Sul - UFMS -Campo Grande (MS), Brasil;Doutora em Enfermagem e Mestre em Enfermagem Obstétrica pela Universidade Federal de São Paulo - UNIFESP São Paulo (SP), Brasil.

2 Professora Adjunta do Departamento de Enfermagem da Universidade Federal de São Paulo - UNIFESP - São Paulo (SP), Brasil; Livre Docente na Saúde da Mulher pela Universidade de São Paulo - USP - São Paulo (SP) Brasil; Doutora em Enfermagem Materno-Infantil pela UNIFESP; Assessora de Programas Institucionais de Educação e Pesquisa da Sociedade Beneficente Israelita Brasileira Albert Einstein. 


\section{INTRODUÇÃO}

O questionamento sobre o conhecimento específico da enfermagem, seus conceitos, seus significados e a utilização desses conceitos na prática, ou seja, a identificação do saber e do fazer da enfermagem que a caracterizam como uma ciência, sempre estiveram presentes em nossa profissão ${ }^{(1)}$.

Desde que Florence Nightingale enfocou as medidas de alívio e de manutenção da higiene, ela diferenciou as ações da enfermeira das do médico, marcando o início da preocupação da enfermagem com sua identidade profissional $^{(2)}$.

Em busca dessa identidade, a enfermagem passou a preocupar-se com o desenvolvimento de um corpo de conhecimentos próprio, que lhe desse legitimidade, visibilidade e autonomia.

A construção do conhecimento reflete não só a elaboração de estratégias específicas para seu desenvolvimento, mas também o amadurecimento dos profissionais ${ }^{(3)}$. O processo de enfermagem constitui então, o mecanismo pelo qual esses conhecimentos são aplicados à prática profissional ${ }^{(2)}$, fundamentando as ações de enfermagem. Dessa forma configuram um conjunto teórico que é a ciência de enfermagem e que é expresso, operacionalmente, pelo processo de enfermagem, o qual busca por meio da sistematização das ações, um nível de qualidade compatível com as necessidades do cliente, de sua família e da comunidade, com os recursos disponíveis ${ }^{(4)}$.

A determinação das intervenções de enfermagem constitui uma das etapas da terceira fase do processo de enfermagem, ou seja, o planejamento da assistência. Assim como ocorre com os diagnósticos de enfermagem e a variada terminologia utilizada para expressá-los, há diversas nomenclaturas empregadas para descrever as condutas das enfermeiras na prática assistencial. Tanto no Brasil quanto internacionalmente, prescrições, ações, intervenções, atividades, condutas e tratamentos de enfermagem têm sido utilizados como termos possuidores de um mesmo significado.

No Brasil, a tendência tem sido para utilização do termo prescrição de enfermagem desde que Wanda Horta introduziu em nosso meio, na década de 70 , o processo de enfermagem. Neste estudo optamos pela utilização do termo intervenção de enfermagem, que segundo a CIPE®(5) é a ação realizada em resposta a um diagnóstico de enfermagem com o objetivo de alcançar um resultado.

A realização da consulta de enfermagem pressupõe necessariamente a aplicação do processo de enfermagem, que permite que a enfermeira determine os diagnósticos de enfermagem e as intervenções de enfermagem correspondentes. A consulta de enfermagem é um procedimento da assistência desenvolvido com base em princípios ou pressupostos teórico-filosóficos definidos, empregando metodologia própria e que, em nosso meio, constitui a aplicação do processo de enfermagem ${ }^{(6)}$. Dessa forma, segundo os mesmos autores, não podemos considerar a consulta como atendimentos ou orientações realizados em corredores.

Designar por consultas de enfermagem ${ }^{(7)}$ quaisquer atividades exercidas pelas enfermeiras, sem fundamentálas cientificamente, é impedir a percepção de sua identidade profissional e empobrecer seu trabalho. A consulta de enfermagem vem sendo considerada como umas das atividades principais desenvolvidas pelas enfermeiras ${ }^{(8)}$, tanto na rede de atenção primária à saúde como na hospitalar, sendo que na rede primária são inúmeras as atividades de prevenção realizadas.

Apesar de estar respaldada legalmente pela Lei do Exercício Profissional $\mathrm{n}^{\circ} 7498$, que atribui privativamente ao enfermeiro a consulta de enfermagem, esta ainda não se encontra incorporada ao processo de trabalho da enfermeira $^{(9)}$, não tendo se tornado rotina nas unidades de saúde $^{(10)}$. Uma das dificuldades para a realização dessa atividade é a falta de conhecimento teórico das enfermeiras que as direcione a um raciocínio e julgamento para a resolução de um problema ${ }^{(11)}$. Portanto, esforços devem ser envidados, desde a graduação, para que esta prática seja realizada com conhecimento científico e competência técnica.

A assistência à saúde da mulher sempre esteve intimamente relacionada com seu ciclo reprodutivo. As consultas de enfermagem, desde que começaram a ser implantadas, sempre foram direcionadas às gestantes. Vargens e Hood ${ }^{(12)}$ expressam sua preocupação com a mulher não-grávida, afirmando que, muito embora os problemas obstétricos sejam graves em nosso meio, o ciclo gravídico-puerperal ocupa um curto espaço de tempo na vida da mulher.

Desde a criação do Programa de Assistência Integral à Saúde da Mulher pelo Ministério da Saúde, em 1984, as informações disponibilizadas referentes ao perfil dessa população relacionam-se ao ciclo gravídico-puerperal, dificultando um diagnóstico de saúde da mulher no que tange aos aspectos não-reprodutivos ${ }^{(13)}$.

Dessa forma, sentimos motivação para orientar nossas atividades assistenciais e de pesquisa à mulher em ginecologia, por concordarmos que a restrição ao enfoque gravídico-puerperal não mais responde às necessidades dessa população.

\section{OBJETIVOS}

- Estabelecer as intervenções de enfermagem e os resultados esperados para os diagnósticos de enfermagem mais freqüentes na assistência à mulher. 
- Construir as intervenções a partir dos eixos A (tipo de ação), B (alvo), C (meios) e H (beneficiário) da Classificação Internacional para a Prática de Enfermagem $\left(\mathrm{CIPE}^{\circledR}\right)$ - versão beta 2.

\section{MATERIAL E MÉTODO}

Trata-se de um estudo descritivo, analítico, retrospectivo, sobre as intervenções de enfermagem estabelecidas para os cinco diagnósticos mais freqüentes em ginecologia, e sobre a construção destas intervenções a partir dos eixos A (tipo de ação), B (alvo), C (meios) e H (beneficiário) da Classificação Internacional para a Prática de Enfermagem $\left(\mathrm{CIPE}^{\circledR}\right)$ - versão beta 2 .

Utilizamos a CIPE ${ }^{\circledR}$ - versão beta 2 como referencial teórico-metodológico, considerando que o critério utilizado pelo Conselho Internacional de Enfermeiras (CIE), para a referida classificação, permite o uso de mais de um modelo teórico de enfermagem, combina diferentes eixos e expressa os conceitos da prática de enfermagem incluindo o vocabulário dos sistemas de classificação existentes como a NANDA, HCC, entre outros ${ }^{(14)}$.
Foram analisados 165 protocolos das consultas de enfermagem realizadas pela autora no Centro regional Dr. Germano de Barros de Souza e no Centro de Atenção Integral à Saúde da Mulher, ambos no município de Campo Grande, estado de Mato Grosso do Sul.

A coleta de dados foi realizada nos registros feitos nos protocolos de consultas realizadas pela autora, após aprovação do Comitê de Ética em Pesquisa e da Diretoria Administrativa das instituições envolvidas.

\section{RESULTADOS E DISCUSSÃO}

Em trabalho científico anterior realizado nos mesmos serviços foram identificados 60 diagnósticos de enfermagem, cuja freqüência simples variou de um a 137, dos quais selecionamos os cinco mais freqüentes para o estabelecimento das intervenções de enfermagem $^{(15)}$. Os cinco diagnósticos de enfermagem mais freqüentes e os resultados esperados foram:

\section{1- Risco para Infecção}

\begin{tabular}{|l|l|}
\hline \multicolumn{1}{|c|}{ FATOR DE RISCO } & \multicolumn{1}{c|}{ RESULTADOS ESPERADOS } \\
\hline início precoce da vida sexual ativa & $\begin{array}{l}\text { A cliente demonstrará comportamento preventivo em saúde } \\
\text { evidenciado pela verbalização de práticas sexuais seguras. }\end{array}$ \\
\hline
\end{tabular}

\section{2 - Risco para infecção}

\begin{tabular}{|l|l|}
\hline \multicolumn{1}{|c|}{ FATOR DE RISCO } & \multicolumn{1}{c|}{ RESULTADOS ESPERADOS } \\
\hline $\begin{array}{l}\text { Vida sexual ativa há mais de cinco anos, associada a uma única } \\
\text { realização do exame preventivo ou a sua não-realização }\end{array}$ & $\begin{array}{l}\text { A cliente comparecerá ao centro de saúde periodicamente } \\
\text { (anualmente) para realização do exame preventivo, mesmo na } \\
\text { ausência de sinais e sintomas associados a problemas } \\
\text { ginecológicos. } \\
\text { A cliente descreverá a finalidade do exame preventivo }\end{array}$ \\
\hline Realização do último exame preventivo há mais de dois anos & $\begin{array}{l}\text { A cliente comparecerá ao centro de saúde na data prevista } \\
\text { (comunicada) para obter o resultado do exame preventivo }\end{array}$ \\
\hline $\begin{array}{l}\text { Falta de conhecimento sobre a importância do retorno à } \\
\text { instituição para avaliação do resultado do exame preventivo e } \\
\text { realização do tratamento adequado }\end{array}$ & $\begin{array}{l}\text { A cliente agendará e comparecerá à nova consulta de } \\
\text { enfermagem ou médica para avaliação do resultado do exame } \\
\text { de Papanicolau }\end{array}$ \\
\hline
\end{tabular}

\section{3 - Comportamento de busca da saúde}

\begin{tabular}{|l|l|l|}
\hline \multicolumn{1}{|c|}{ CARACTERÍSTICA DEFINIDORA } & \multicolumn{1}{|c|}{ FATORES RELACIONADOS } & \multicolumn{1}{c|}{ RESULTADOS ESPERADOS } \\
\hline $\begin{array}{l}\text { Desejo expresso ou observado de procurar } \\
\text { informações para a promoção da saúde }\end{array}$ & $\begin{array}{l}\text { Relacionado à procura do centro de saúde } \\
\text { para realizar o exame preventivo, para } \\
\text { realizar tratamento para hipertensão } \\
\text { arterial, realização do auto-exame de } \\
\text { mamas, abandono do hábito de fumar }\end{array}$ & $\begin{array}{l}\text { A cliente permanecerá apresentando } \\
\text { compomonto de prevenção de drogas e } \\
\text { mais, elevar seu nível de saúde recorrendo } \\
\text { aos serviços oferecidos à população }\end{array}$ \\
\hline
\end{tabular}




\section{4-Conhecimento deficiente}

\begin{tabular}{|c|c|c|}
\hline CARACTERÍSTICAS DEFINIDORAS & FATORES RELACIONADOS & RESULTADOS ESPERADOS \\
\hline $\begin{array}{l}\text { Verbaliza a deficiência de conhecimento ou } \\
\text { habilidade; solicita informação; expressa } \\
\text { percepção incorreta acerca do estado de } \\
\text { saúde; não desempenha corretamente um } \\
\text { comportamento de saúde prescrito ou } \\
\text { desejado }\end{array}$ & $\begin{array}{l}\text { Relacionado à necessidade de retorno à } \\
\text { instituição onde realizou o preventivo para } \\
\text { tomar conhecimento do resultado } \\
\text { Relacionado à anatomia e fisiologia do } \\
\text { aparelho reprodutor feminino } \\
\text { Relacionado ao auto-exame das mamas } \\
\text { Relacionado à aplicação de creme vaginal } \\
\text { Relacionado aos métodos contraceptivos }\end{array}$ & 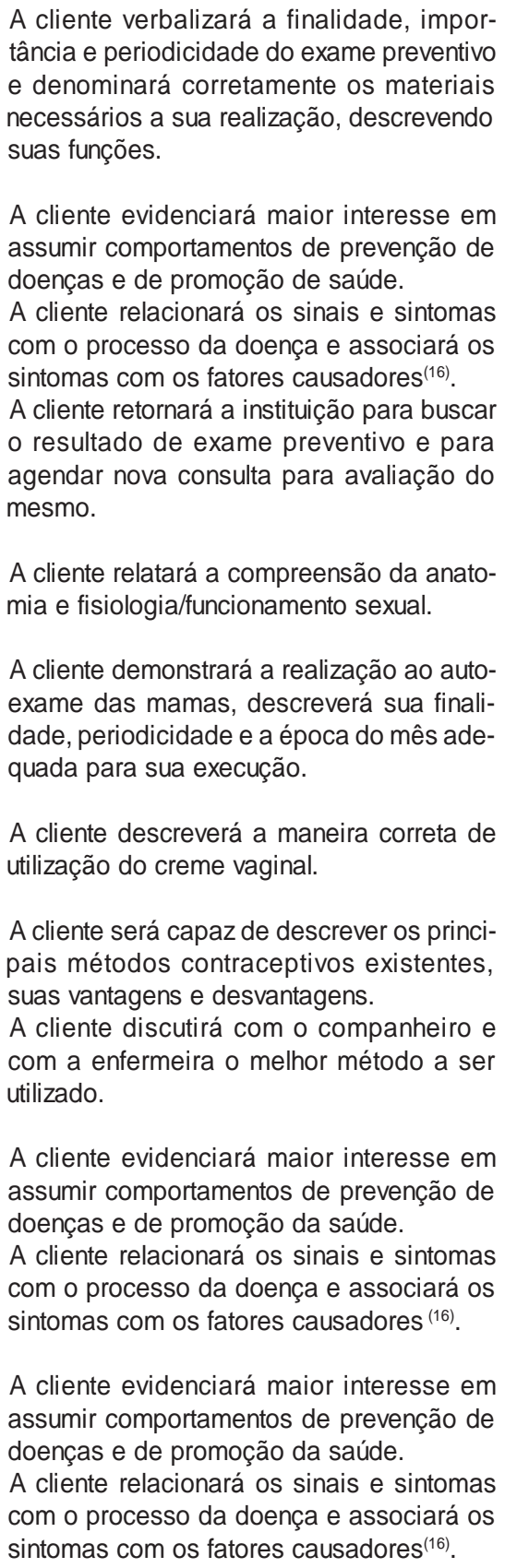 \\
\hline
\end{tabular}

A assistência de enfermagem foi planejada com base nos resultados esperados, e as intervenções foram elaboradas em linguagem natural nos protocolos das pacientes e constituíram a base de dados necessária para a construção das intervenções de acordo com a classificação da CIPEâ - versão beta $2^{(5)}$ e que estão explicitadas nos quadros $1,2,3,4$ e 5 . 


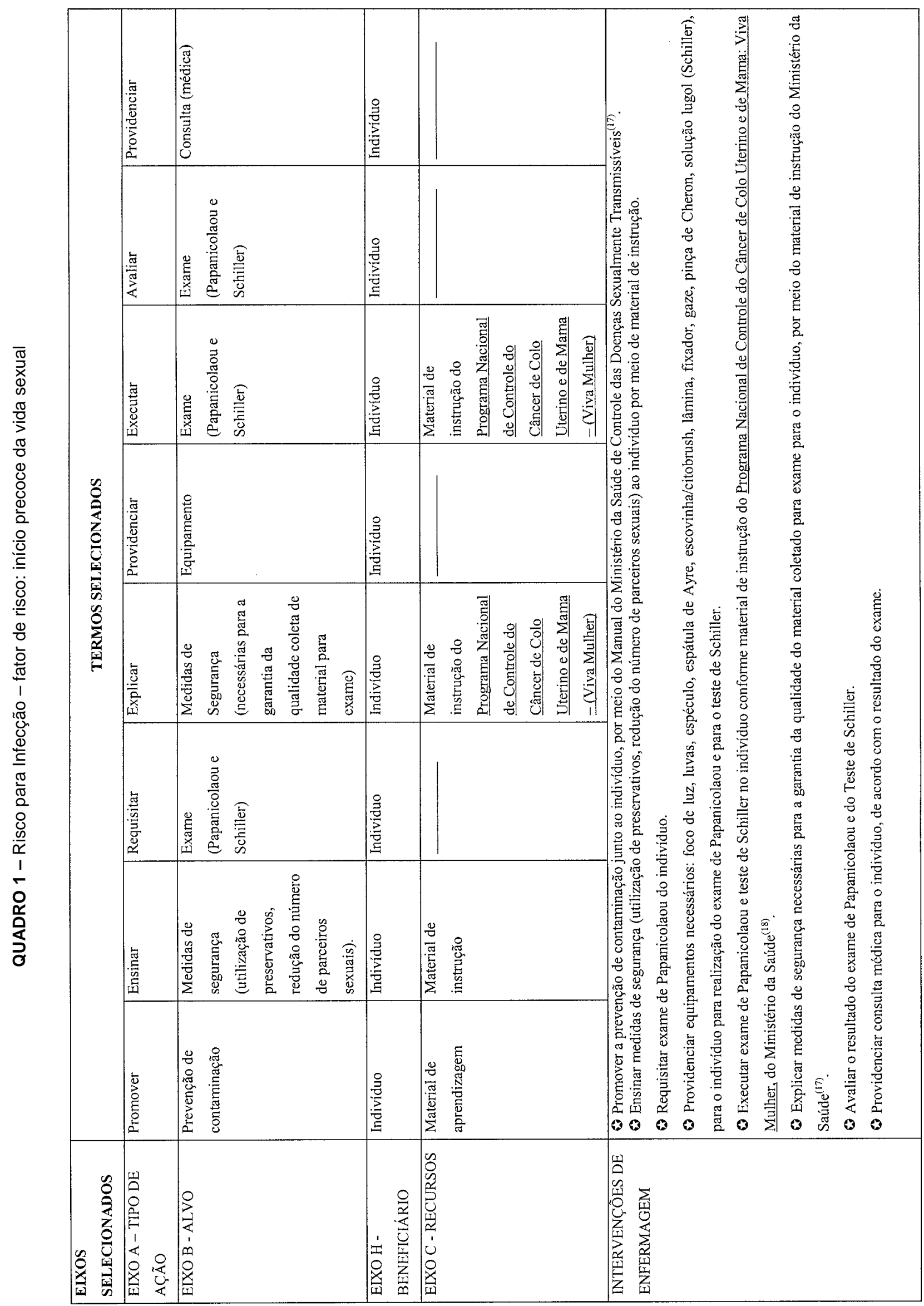




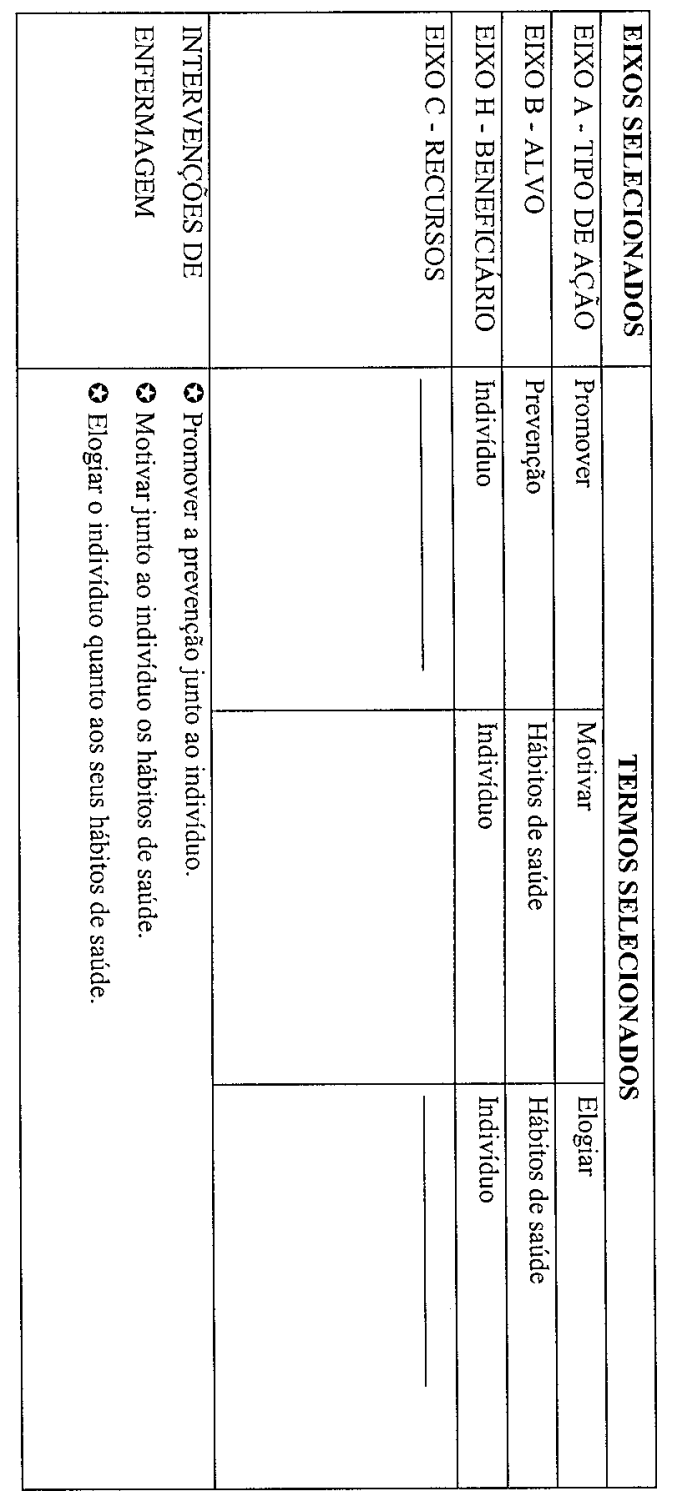

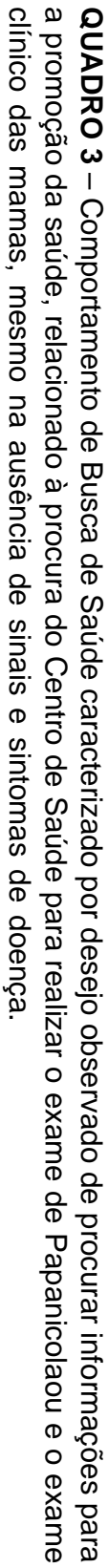

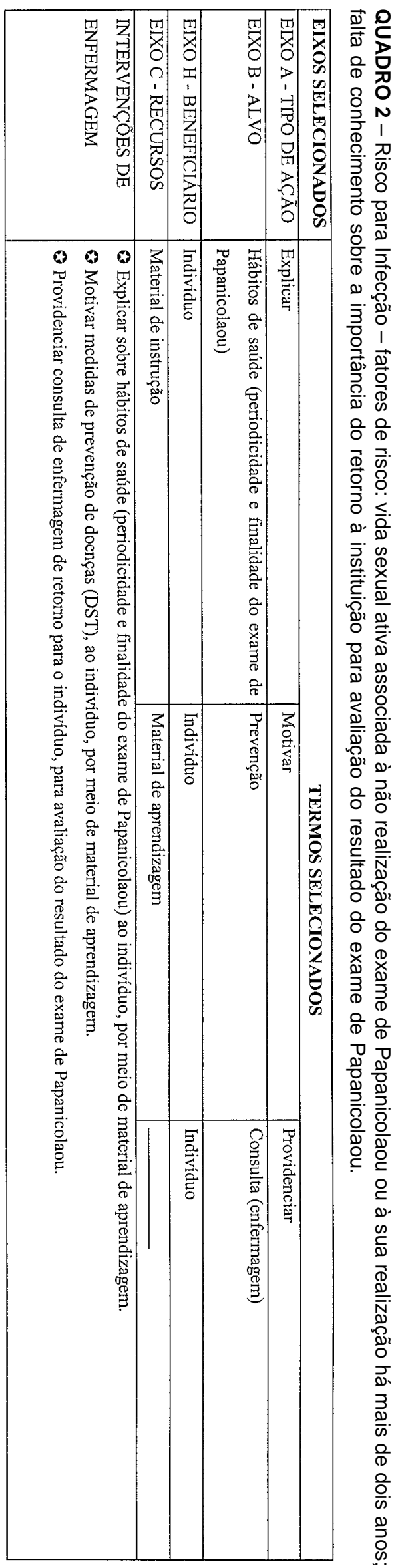




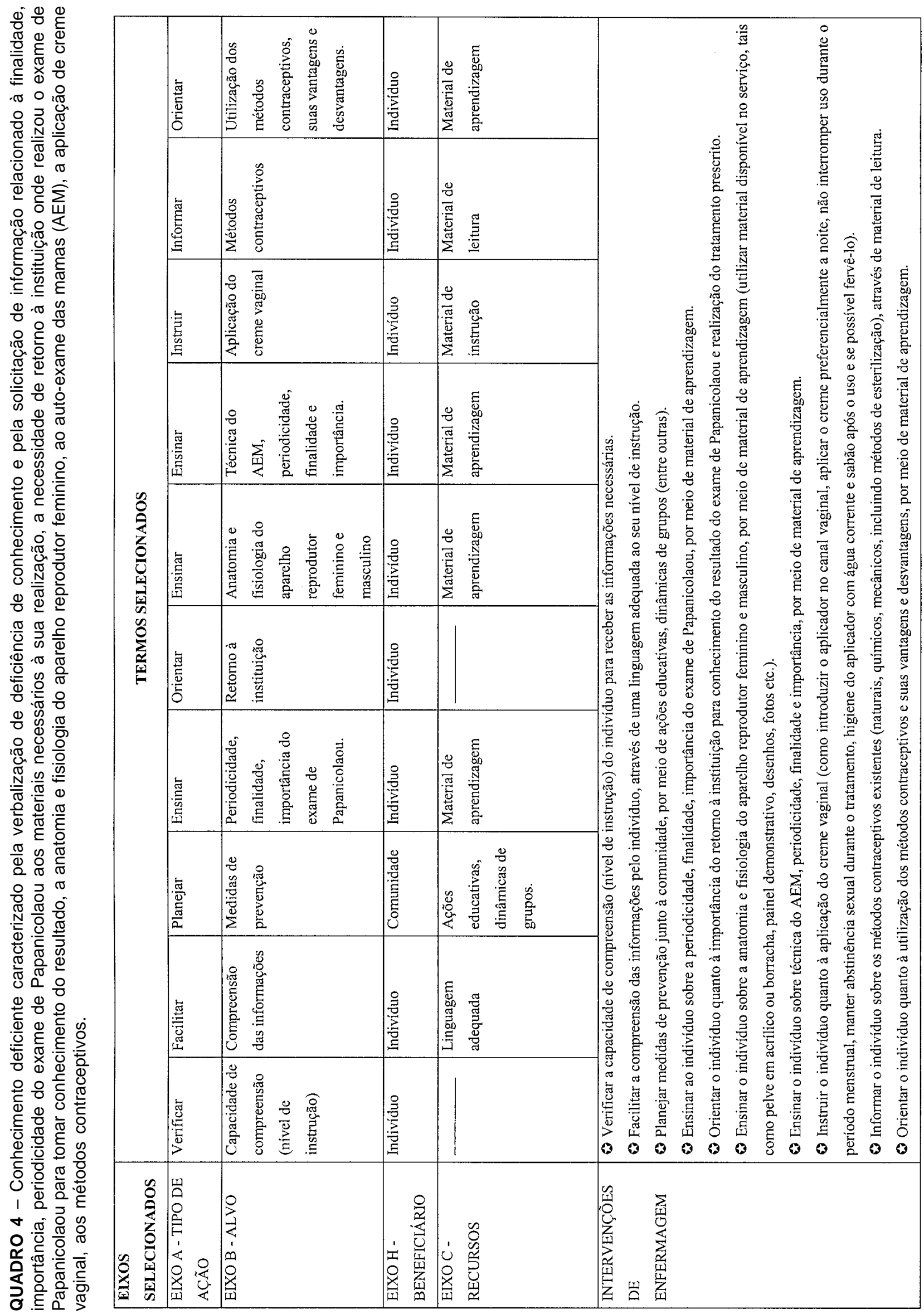




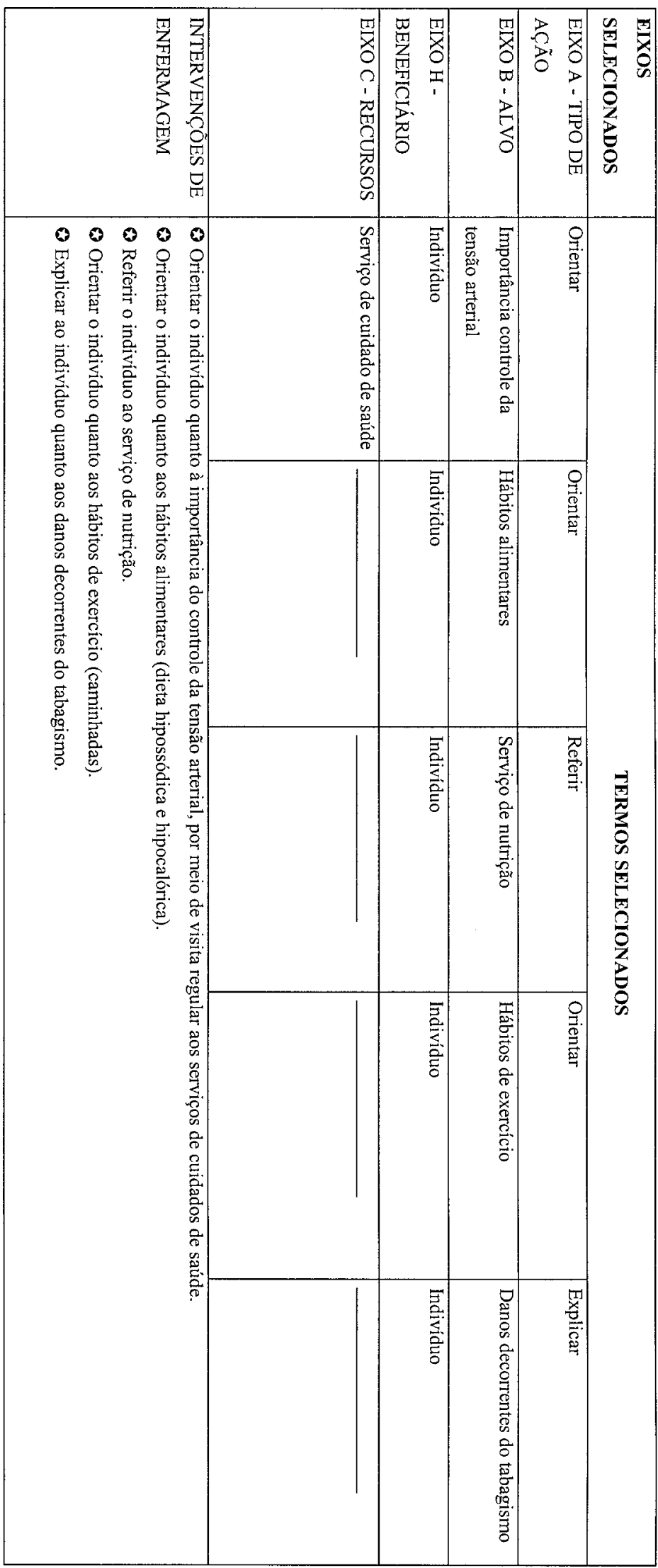

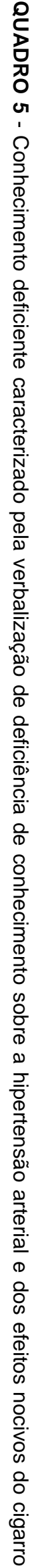




\section{CONCLUSÕES}

No presente trabalho, construímos as intervenções de enfermagem a partir da classificação proposta pelo CIE - a CIPE ${ }^{\circledR}$, versão beta 2- e para tanto foi imprescindível a utilização da linguagem natural.

Por serem escassas as pesquisas na área de enfermagem em ginecologia, principalmente no que diz respeito à assistência à mulher em Unidades Básicas de Saúde, as tarefas de determinar e construir as intervenções de enfermagem, a partir da prática em consulta de enfermagem, revelaram-se bastante complexas.

Dos eixos que compõem as intervenções de enfermagem segundo a CIPE $^{\circledR}$ - versão beta 2 , foram selecionados os eixos A (tipo de ação), B (alvo), C (meios) e H (beneficiário). Destes, apenas o eixo A continha a correspondência exata com a linguagem natural. Para os demais eixos, foi necessária a inclusão de termos nãoexistentes na CIPE $^{\circledR}$, para que as intervenções pudessem expressar claramente a intenção da ação.

As ações mais freqüentes foram 'orientar' $(\mathrm{n}=30)$, 'referir' $(\mathrm{n}=29)$, 'explicar' $(\mathrm{n}=27)$, 'prover' $(\mathrm{n}=25)$, seguidas por 'aconselhar' $(\mathrm{n}=16)$, 'desempenhar' $(\mathrm{n}=$ 16), 'motivar' $(\mathrm{n}=16)$, 'verificar' $(\mathrm{n}=15)$, 'avaliar' $(\mathrm{n}=$ 14), 'promover' $(n=13)$, 'requisitar' $(n=13)$, 'ensinar' $(n$ $=10)$, 'instruir' $(\mathrm{n}=10)$, 'informar' $(\mathrm{n}=10)$, 'estimular' $(\mathrm{n}=10)$, 'reforçar' $(\mathrm{n}=8)$, 'estabelecer relação com' $(\mathrm{n}=$ 4), 'facilitar' $(n=3)$, 'escutar' $(n=3)$, 'examinar' $(n=2)$ e 'tratar' $(n=2)$. As demais, com ocorrência $n=1$ foram 'apoiar', 'otimizar', 'definir o perfil', 'triar', 'confortar', 'segurar a mão', 'inspecionar', 'elogiar' e 'planejar'.

Ao determinarmos as intervenções, verificamos a necessidade premente de trabalho multidisciplinar, uma vez que em nossa pesquisa a ação referir mostrou-se como equivalente ao encaminhamento a outros serviços de saúde, como o de nutrição, o de psicologia, o serviço social e o serviço médico, entre outros.

Outra constatação foi a imprescindibilidade do papel de educadora da enfermeira. De fato, embora saibamos que esse papel não se restringe apenas à transmissão de conhecimentos, ele aqui se evidenciou por meio de diferentes ações: 'orientar', 'explicar', 'aconselhar', 'motivar', 'promover', 'ensinar', 'instruir' e 'informar'.

Estamos cientes de que, o desenvolvimento do estudo em duas instituições públicas de saúde é um fator limitante, devido à complexidade do tema. Porém oferecemos elementos produtivos para que novas pesquisas sejam empreendidas na área de assistência de enfermagem à saúde da mulher, com foco na elaboração do plano de cuidados padronizado. De nossa parte, pretendemos dar continuidade a este trabalho em duas vertentes: utilizando uma classificação que permita lidar com os resultados esperados; por outro, validar clinicamente as intervenções de enfermagem.

\section{REFERÊNCIAS}

1. Nóbrega MML, Gutiérrez MGR. Introdução. In: Nóbrega M ML, Gutiérrez MGR. Equivalência semântica da classificação de fenômenos de enfermagem da CIPE: versão alfa. João Pessoa: Idéia; 2000. p.13-23.

2. Rossi LA, Casagrande LDR. Processo de enfermagem: a ideologia da rotina e a utopia do cuidado individualizado. In: Cianciarullo TI, Gualda DMR, Melleiro MM, Anabuki MH. Sistema de Assistência de Enfermagem: evolução e tendências. São Paulo: Ícone; 2001. p. 41-62.

3. Cianciarullo TI. O desenvolvimento do conhecimento na enfermagem: padrões de conhecimento e sua importância para o cuidar. In.: Cianciarullo TI, Gualda DMR, Melleiro MM, Anabuki MH. Sistema de Assistência de Enfermagem: evolução e tendências. São Paulo: Ícone; 2001. p. 41-62.

4. Cianciarullo TI. Teoria e prática em auditoria de cuidados. São Paulo: Ícone; 1997. A qualidade na enfermagem; p.15-20.

5. Conselho Internacional de Enfermagem (CIE). Classificação internacional para a prática de enfermagem beta 2 . Traduzido de Marin FH. Genebra; 2003.

6. Vargens OMC, Araújo LM. Consulta de enfermagem ginecológica: relatando uma experiência. Rev Enferm UERJ. 1997; 5(1):367-72.

7. Car MR, Egry EY. Processo de trabalho da enfermagem na atenção ambulatorial ao adulto com hipertensão arterial: representações a contradições. Rev Esc Enferm USP. 1995;29(2):180-92.

8. Melleiro MM. A consulta de enfermagem no cenário do sistema de assistência de enfermagem. In: Cianciarullo TI, Gualda DMR, Melleiro MM, Anabuki MH. Sistema de Assistência de Enfermagem: evolução e tendências. São Paulo: Ícone; 2001.p.279-92.

9. Henriques RLM. A visão dos enfermeiros sobre a consulta de enfermagem. Rev Enferm UERJ. 1993;1(2):31-8.

10. Gomes ML. Quando são mulheres (clientes e enfermeira) na consulta de enfermagem. Rev Enfermagem UERJ. 1996; (ed. extra):109-12.

11. Castilho V. A enfermeira vivenciando a formulação de conduta de enfermagem [tese]. São Paulo(SP): Escola de Enfermagem da USP; 1991.

12. Vargens OMC, Hood MD. E a mulher não grávida? Rev Enferm UERJ. 1993;1(2):96.

13. Rocha CR, Siqueira PRA, Oliveira PRF, Moura MAV, Spindola T. A enfermagem e a saúde da mulher: questões de gênero e sócio-políticas. Esc Anna Nery Rev Enferm. 2000;4(1):105-14.

14. Nielsen GH, Mortensen RA. The architecture of ICNP: time of outcomes. Part I. Int Nurs Rev. 1997;44(6):182-8.

15. Gerk MAS. Conjunto prioritário de diagnósticos de enfermagem em ginecologia [tese]. São Paulo: Departamento de Enfermagem da UNIFESP; 2000.

16. Doenges ME, Moorhouse MF. Diagnóstico e intervenção de enfermagem. 5a ed. Porto Alegre: Artmed; 1999.

17. Ministério da Saúde. Secretaria de Políticas de Saúde. Coordenação Nacional de DST e AIDS. Manual de controle das doenças sexualmente transmissíveis - DST. $3^{\text {a }}$ ed. Brasília; 1999.

18. Instituto Nacional do Câncer (INCA). Programa Nacional de Controle do Câncer do Colo do Útero e de Mama: Viva Mulher. [texto na internet]. Brasília; 2003. Disponível em: http://www. inca.gov.br/prevencao/programas/pnccum/_(20 maio 2003). 